\title{
"To See a World in a Grain of Sand": Complexity Ethics and Moral Education
}

\author{
HEESOON BAI \& HARTLEY BANACK \\ Simon Fraser University (Canada)
}

\begin{abstract}
Making the case for the mutual relationship between ontology (what reality is like) and ethics (how we should conduct ourselves), this essay argues that the dualistic, linear, deterministic ontology of Modern Science that categorically separates perceiver and the perceived, knower and known is oppressive by virtue of objectification. Delineating a relational (that is, non-linear) ontology disclosed by New Science and Complexity Theory, this essay extrapolates to an ethical paradigm, named 'participatory ethics'. Key to participatory ethics is perception of "patterns that connect" - which, to manifest, the moral agent needs to emergently embed itself in the pattern, in a manner analogous to fractal reiteration. Since non-linearity (complexity) manifests everywhere we turn and in everything we encounter, participatory ethics modeled after complexity is about recalling, remembering, and reminding ourselves of, our inter-beingness.
\end{abstract}

\section{Introduction}

The birds have vanished into the sky, and now the last cloud drains away. We sit together, the mountain and me, until only the mountain remains. (Li Bai, 701-762)

Complexity as a mathematical-scientific notion is interesting to us, for it seems to capture with its rich conceptual vocabulary something we sense in living: flow, embededness, spontaneity, creativity, and contextuality. 
What does an ethic in resonance with this sense of living look, feel, and taste like? This article is about ethics seen in the light of complexity theory. From the outset, we shall be careful here, though. We are not proposing to add yet another normative moral theory-and pursuant theory of moral education-to the current proliferation of theories prescribing norms of disposition and conduct, as in moral rules, laws, and imperatives. ${ }^{1}$ Nor are we confining our meanandering thoughts to any complexity theory. Certainly, we too, will indicate paths or ways, but more attuned to Dao (道, the Way), where 'way' shall be envisioned as patterned images found in the most marvelously intricate folds of fractals. A perception of Dao, or the way of complexity, emerges from "slow" seeing and attentively observing the phenomenal world in which we are intimately embedded. Reminding and remembering will enhance this seeing and sensing, which henceforth shall be called participatory ethics, accenting ethos ${ }^{2}$ in "ethics" as a way of communal life. It will be of this recalling and remembering that ethics, congruent with complex ways of co-emergence, may illuminate thought, conversation and action. Along the way, we may also catch some new insights into education that this ethical perspective allow us; that is, if we are attentive and receptive. We believe that in meditating upon, speaking about, and acting on this recollection of way, so integral to our being, creativity may ensue. Creativity has the power to respond to trends of harmful habits of mind-heart, such as control, manipulation, and exploitation-essential aspects of modernity fueled by objectifying ideologies that separates matter from mind, self from other, and knower from known. Inspired by complexity theory, our ethical notion, including thoughts on moral education, offers not prescriptive and proscriptive solutions but creativity. Our offering to the reader ${ }^{3}$ may be conceived as a poiesis rather than solutions that, however tempting, exist but as ghosts. Complexity of our being, including inescapable impermanence, precludes absolutes, such as moral imperatives. Instead of prescriptions of absolutes, we recall creativity inherent in our awareness of complexity and celebration of pattern. So let us thread our way cautiously through mazes of moral absolutes tempting us with solutions. Let us tread lightly in hope that we may glean glimpses of fleeting snowflakes falling through the calm of night and resting ever so briefly upon warm skin.

\section{Ethics and Ontology}

Ethics as a study of moral norms and conduct invites us to inquire, imagine, and enact different conceptions of the good life. Thus, living well is the central concern of ethics, and takes priority over considerations of moral rights and wrongs. What is right and wrong is derivative from (and therefore is relative to) particular conceptions of the good life that a given community 
or society has. Historically, there have been varieties of competing and complementing accounts of ethics or morality. For the purposes of this paper 'morality' and 'ethics' will be understood as synonymous. Our intellectual history-and pre-history to some degree-amply demonstrates that humanity has been entertaining a wide array of worldviews since antiquity. ${ }^{4}$ What should follow from this observation is that our moral views must have been changing, too, to reflect our changing worldviews. (We cannot be sure which changes first, but that they mutually inform and mutate seems plausible.) Consider a historical survey of different cultural and societal groups', and their subgroups', changing codes of ethics. For example, it was morally wrong in many cultures to treat women as equals to men. People believed in a natural inferiority of women to men. People even believed that women could not be as morally developed and competent as men. ${ }^{5}$ In many enclaves of the world, such beliefs still hold. Or consider the status of homosexual unions from culture to culture nowadays. Yet another example: Many who believe in the immortality of the soul often see the reason for doing good and being good in terms of consequences their actions have on their afterlives. Atheists and annihilists would have no such options. Examples abound. The central point we want to make with these observations is that the substantive views of how we should conduct ourselves to live a good life (ethics) are predicated upon our understanding of what the world is like (ontology) and what humans are like (philosophical anthropology). Our understanding of world and human nature changes and varies across culture, geography, and time; so do our ethical orientation and paradigms.

We are interested in exploring the pictures of reality that New Science, such as quantum physics, chaotic dynamics and complexity, present, and noting how these pictures may reshape our ethical vision and understanding -in short, ethical paradigms and orientations. In this paper, we shall make the case that the ontology of the Mechanical Universe (Newtonian Science) that 17th-century Science and Philosophy championed, lends itself easily to the operations of oppression such as control, domination, and exploitation. We shall assert that the ontology of the Mechanical Universe with its dualism, reductionism, essentialism, and determinism disposes us to moral fundamentalism by virtue of objectivist language, imposing categorical and linear thinking as absolutes. Absolutes are at home in a deterministic universe governed by linear causality. Complexity theory contests this picture of the world and our place in it.

What would an ethic infused with complexity theory look like? How would we approach good life and moral conduct differently if we acted out of an ethic of complexity? What are the implications of such an approach for ethical education and schooling? With these questions in mind, we now turn to an exploration of the contrasting ontologies presented by Classical 
Science (meaning, Modern Science) and New Science. Our exploration will have to be selective, for we are mainly interested in ethical implications of central New Science concepts. Therefore, we will not treat theoretical discussions of New Science in this paper.

\section{Mechanical Universe and Oppression}

Today, we are living the legacy of a scientific worldview that has been in the making since pre-Socratic atomists in Ancient Greece first espoused the theory of atoms and that received a definitive philosophical justification and practical application in $17^{\text {th }}$-century Europe. We refer to the inauguration of Modernity by philosophers and scientists like Hobbes, Descartes, Galileo, and Bacon. ${ }^{6}$ These thinkers proffered us, by philosophical argument and scientific analysis, a mechanical universe composed of inanimate matter and strictly ruled by linear causality. The essential feature of this deterministic universe is extension-space occupied by matter. Whatever is not part of this world of extension belongs to the unreality of intention-mere subjective illusions having no "real"; that is, measurable and objective value.

The metaphysical context for the Mechanical Universe is dualism-the ontological belief that two worlds exist: one is an objective world of matter having the characteristics of extension, the other a subjective world of perception involving intention and attention. Cartesian Dualism, recognizing two distinct and separate "substances," the mental and the physical, is a clear example of this ontology. There, physical entities exist independent of subjective experiences like sense perceptions, emotions, intentions, and values. In everyday life, we often do not maintain a strict separation between the objective and the subjective, and are prone to attributing subjective to objective. In scientific circles, this sort of thinking is scornfully called primitive, magical, or animistic. Scientific training supposedly trains us to abandon such thinking. This consists of abstracting subjective data from objects: what Laing (1980) calls "the ablation or elimination of some or all sense data," for the goal of "the de-realization of any subjectivity out there" (p. 9). ${ }^{7}$ Perfect objects are uncontaminated by any subjectivity, and thus are free of human feelings and values, including positive ones like compassion, love, and respect. In other words, perfect objects are inanimate: dumb, mute, insentient and permanent. Since they are devoid of feelings and values, we are liberated to act capriciously with them. We control, use, exploit, and destroy them however we wish. In the same passage that speaks of the ablation of all sense data, Laing reminds us that this process of objectification is what we do to others, and that objects as such are not givens in nature. Thus, we can render anything or anyone, including fellow human beings, into an object through a process of eliminating or denying any subjective 
sense to their reality. It is this psychological process of objectification that makes, for example, genocide conceivable.

Many signs suggest that the world we have created according to the premises of the Mechanical Universe is ecologically deteriorating, socially disintegrating, economically fraying, and psychically fractured. Mounting sentiment of despair and dread, while sometimes paralizing, is more often than not providing pervasive energy to shifting or broadening of the hegemonic picture of the universe. Such shifting has been occuring for some time now. Whether it comes under the name of cybernetics, systems theory, quantum mechanics, or chaos and complexity theory, names aside, all signal an end to a certain ontological picture of the world; namely, the Mechanical Universe, that has guided and limited perception, thinking, and consequent action for a few centuries.

\section{Relational Universe and Respons-ability}

Let us now turn to the "new science" of quantum mechanics and chaos dynamics and its challenges to the deterministic, linear, and dualistic or discontinuous view of the world. ${ }^{8}$ We shall not go into technical details of these scientific and mathematical theories but posit some main concepts that these theories proffer and consider the implications for ethics.

Quantum Physics has challenged the traditional separation between, therefore the dualism of, perceiver and perceived, subject (self) and object (other), and knower and known. ${ }^{9}$ In the quantum world, the externalizing viewpoint that we conventionally assume towards the world "out there," towards "objects," vanishes. If " $\mathrm{I}$ " is embedded in "it", so that my perception is part and parcel of the phenomenal reality " $\mathrm{I}$ " observe, how can we go on speaking of the world "out there" as if "I" am not "over there" but only "in here"? How could we draw an impermeable distinction and separation between what is "out there" (objects observed) and what is "in here" (perceptions)? As well, when there is constant interchange of matter, form, and structure amongst all that exists, so that they undergo constant change in composition, identity, and disposition, how is it that we perceive and treat objects as if they are discrete, bound, substantive, and self-same? Thus the quantum-mechanics worldview challenges the notion of linear causality that assumes discrete entities and their extrinsic relationships.

The universe that quantum physics and chaotic dynamics reveal is a fundamentally relational universe where subject and object are interpenetrating presences. In a relational universe, to speak of 'an object' is to speak in a short-handed way of patterns of complex, dynamically interpenetrating relationships. These relationships are dynamic and non-linear. The relational universe is not the world of discrete, atomistic objects that behave determinis- 
tically, therefore predictably, according to linear causality. As A interacts with $B$, the identity of A changes to reflect its interaction with B. Likewise for B. As $A$ and $B$ continue to interact, along with innumerable others, changes to their "identity" complexify beyond measure and prediction. When things undergo continuous transformations, it is misleading to speak of them as having relationships, as if there were these self-same, permanent objects whose interactions with the world produce external, circumstantial changes to the objects. This impression is deceptive. Relational changes are internal, affecting the very identity of a given thing, to the point that it is indeed misleading to speak of "identity." It is more apt to speak of "multiplicity," or better yet, "complexity." All of us, including non-human beings, are our relationships. We are nothing other than our relationships-with each other, with the world. We are patterns that connect. This does not mean that we cannot speak of individual forms and objects, but their usual substantive and reified connotations have to go. Forms and objects are the in-the-moment temporal freezing of flowing patterns. In this understanding, then, we can think of form and pattern in the same way that we think of light being wave and particle at the same instant.

Phenomenologically we experience ourselves and the world as one-a singular. Varela traces this to the work of Merleau-Ponty and states that "the organism both initiates, and is shaped by, the environment, [so that] we must see the organism and environment as bound together in reciprocal specification and selection." ${ }^{10}$ However, by postulating, as we conventionally do, simple and tidy reified objects in addition to complex and messy phenomenological processes, our attention shifts from the latter to the former. This is what 'objectification' does. When objectification happens, what we get is an 'I-it' relationship that psychologically disposes us to degrees of oppression. ${ }^{11}$

How does inhabiting a relational universe inform our ethical understanding and practices? And, what subsequent intimations may inform education and schooling? For example, today's educational discourse is dominated by the theme of social responsibility. How would we do our social responsibility differently if we were to inhabit a relational universe? Traditionally, there have been two ways of looking at responsibility: deontology and naturalism. In deontology (in Greek, deon means duties and obligations) we go by prescribed and proscribed rules and principles. Inclinations and desire figure little or not at all in deontology. To be socially responsible means for individuals to know what their obligations and responsibilities are and then go ahead and fulfil them, notwithstanding what their inclinations and disinclinations may be. In naturalism, we make ethical decisions, which are influenced or even formed by perceptions, inclinations, and desires. ${ }^{12}$ Our ethical conduct flows out of such perceptions and feelings. Thus, to be responsible for something or someone is to respond to the other's needs out 
of care, compassion, love, and so on. Psychologically speaking, these otherregarding virtues or dispositions emerge from the realization of one's deep interconnection with the other. One sees that one's reality is embedded in the other's. One is implicated in the other. From this embeddedness, one perceives, senses, and feels the other's reality, even if vicariously, and naturally and intuitively responds to it. Or, to put it more epistemically, one's knowing what the other's reality is like, and knowing how to respond appropriately to the other, is a function of realizing ${ }^{13}$ one's reality being embedded in the other's. In other words, naturalistically "felt" responsibility comes out of the realization that one is inhabiting a relational universe.

Now, the practical question that we must always ask as educators is: How do we support ourselves and others to come to this realization? This is where we look to complexity theory once again for further illumination and articulation. Employing a number of concepts in this theory, we can articulate an ethical paradigm that realizes a relational universe.

\section{Non-linearity, Pattern Perception, and Participatory Ethics}

The Relational Universe is a complex system because it is not solely (if ever) linear. Linear causality operates in closed, deterministic systems composed of discreet entities interacting adventitiously, hence, predictably. Theoretically - that is, not factoring in human ignorance and imperfections-what happens to anything in such a universe is perfectly predictable, given operations of linear causality. This ideal environment is even difficult for traditional science where replication is fundamental for understanding. How can, for instance, two experiments ever really be absolutely reproduced? And if not, how much difference is acceptable or deemed negligible to maintain intact the overall myth of repetition and thus predictability according to linear causality? New Science recalls that ours is not a linear, deterministic (in the sense we explained in footnote 8 ) universe; hence, fundamentally not predictable. We can only anticipate, but not predict. Yet, a lot of us want a predictable universe, presumably to assuage our sense of deep existential insecurity. Thus we conjure up a mechanical universe so as to live a life of predictability, hence security. To make that happen, we have to adopt a life of control, domination, and conquest. The more we control and conquer something, the more predictable it appears to become, and, presumably, the more secure we would feel. Given this logic, wishing to live in the mode of control, conquest, and domination, requires us to choose a deterministic, linear, therefore, predictable universe, like Plato's universe of Perfect Forms or its mathematized scientific realization as mechanical universe. ${ }^{14}$ Psychology (of existential insecurity) leads ethics (of control and domination as a way of life), and ethics leads ontology (of the Mechanical Universe). Hence, 
psychology leads ontology. We imagine the universe (and think real) according to the feel of the kind of life-world (Lebenswelt) we inhabit. ${ }^{15}$

We wish to recall that we are living in a non-linear universe where uncertainties, surprises and creativity are determining conditions contained in, and emergent of, what has happened. ${ }^{16}$ Non-linearity is a property of an open system "far from equilibrium," to use Priogine's phrase, ${ }^{17}$ where contingency and instability characterize the predominant pattern. "Catastrophe bifurcations," forking points of alternate reality and choice, abound. Non-linearity implies impossibility of the necessary and perfect, hence the impossibility of complete prediction, to exist. This does not mean we live in a random universe. Between necessity (that is, predictability) and pure chance (that is, unpredictability), there lies a vast region of creativity: patterns of opportunity and intervention that are not perfectly predictable but not without form. These patterns, though not formulas or templates, and while not amenable to precise calculation, reveal underlying order in terms of our experience of given phenomena. Order here is not something that will ever come under "facts," but rather points to somatic wisdom of being. Think of our grand aphorisms like: "Red skies at night, sailors' delight. Red skies at morning, sailors take warning." This does not give an absolute fact but a heuristic reminder. Another example: you are waiting at a bus stop. Even if the bus is scheduled to arrive at $2 \mathrm{pm}$ and your watch says 2:05 or 2:10, you will not leave, thinking that this event will not happen, now that the determined time has past. Rather, you incorporate this variance into your understanding of the possibilities around this experience. This is how we come to think in patterns, in contrast to, in facts.

A present tendency is still to treat patterns as if they were "facts". We objectify them. It is not just laypersons who operate this way; scientists, mathematicians, and philosophers often slip into this mode. Let us not forget that patterns are not independent of perception, which means that the perceiver's act of perception and interpretation are part of the pattern perceived. To even tease these two apart, for discussion's sake, is non-conducive to the notion implied. Yet, so long as we use conceptual language, we are trapped in the tendency to objectify. ${ }^{18}$ Sensing pattern implies seamless interpenetrating relationships between perceiver and perceived, subject and object. The perceiver experiences patterns by virtue of consciously "becoming one with" the reality in which one is already un- or subconsciously part of. Sayings like "becoming one with" may make pattern perception a super-normal occurrence, but of course we do not mean that. Sensing pattern happens all the time, as it is part of how our cognition is shaped. Yet, this does not mean that we are practiced at it or there is no need for training and improvement. One may have capacity to play piano like a virtuoso, but in actuality, one can only bang out a crude tune, due to lack of exposure, training, and other conditions preventing fluency and creativity. Pattern sensing is analogous. 
Roles of education abound in this context.

Sensing patterns accesses a different kind of cognition from mathematical-logical thinking of linear, analytic operations.$^{19}$ For sensing "patterns that connect," one needs to proceed consensuously-that is, sensuously (and feelingly) embedding oneself in perceived phenomenon. We can perceive patterns that connect, not because there are pre-given patterns ("data"), or facts, but because we become part of, not apart from, emergent patterns of integrated whole-a Gestalt. To know the pattern, one must be part of the pattern. Let this be called "participatory knowing". ${ }^{20}$ To emphasize, the key aspect of participatory knowing, or consensuality, is that the "knowing subject" becomes inscribed in context and thus epistemically intimate with "it." (Again, let us be mindful how the term 'it' tends to trigger objectification.) Here, "object" is reconceived from being something that lies outside subject, "out there," independent of perceiver, to becoming a phenomenon continuous with self, unfolding creatively through the self's perception. Now, the knower is part of what comes to be known, and the observer is part of the observed.

Long-standing comprehension of ethics as a system of prescriptions and proscriptions about how we should or should not act, and how we should or should not be, induces the objectifying, dualistic, and linear consciousness that separates self from other, subject from object, perceiver from perceived, and doer from the deed. Only within the framework of such understanding does it make sense to tell (instruct, enjoin, command) others (and oneself) what to do and how to be, and expect people to change accordingly in behaviour, feelings, and states of being. If such deontological moralizing functioned, after millennia of moralizing, we would have "heaven on earth." Yet, not only does this not work (as far as we can tell), but by way of it, moral fundamentalism is advanced. Morality becomes the central practice of control, suppression, oppression, and domination, resulting in part in power politics of violence. For example, increasing fundamentalism worldwide is threatening world peace. ${ }^{21} \mathrm{At}$ the heart of fundamentalism is a worldview ignorant of, or ignoring, non-duality of self and other, perceiver and perceived, subject and object, and so on. If we think we live in a universe of linear causality, composed of discrete beings whose intentions and movements that self (or, for that matter, God) can control, then we are more likely to move towards ethics and politics of power where subject dominates and manipulates object, resulting in exploitation and violence.

\section{Contours of Participatory Ethics and Moral Education}

The difference between sensing oneself as having relationships and as being relationships has profound ethical and educational implications. In this penultimate section, we shall attempt to outline some of these and present 
them as "principles" of participatory ethics, therefore, by extension, participatory moral education.

\section{Inter-being}

In acting, we inevitably bring about changes in the world. Changes result from our interpenetration with/in the world. In assuming an externalist and objectifying viewpoint, we think of change in terms of what we have to do to the world. This tendency is everywhere, whether we are talking about interpersonal or environmental conduct. We act in terms of doing something to an Other, an object, to fix perceived problems. A teacher sees Johnny as having a problem; worse, Johnny becomes a problem in class. The inclination to externalize (in psychological terms, to project) our problems-that is, sensing problems as being out there, residing with others-predisposes us to interact with the world in the mode of control and manipulation. The latter is what seems to happen when the self-other dichotomy is set up, and self senses other as having a problem or even being the problem. Rules, maxims, regulations, and policies are often imposed on others (and ourselves) to control and manipulate behaviour. ${ }^{22}$ Hence the emergence of the social contract. ${ }^{23}$ In participatory ethics, however, other is sensed as interpenetrating matrices of relationships, thus forming inter-being ${ }^{24}$ with self, and self is contemplated likewise; consequently we think, speak and act not in terms of control but of recognizing and tending to relationships between self and other.

As educators, we have the possibility to impart a participatory sense of being to our students. What might forms of participatory education be like? To begin with, teacher as explicator would not occur, for teacher would understand that teaching does not occur apart from student learning, binding teaching and learning to be the mutual experience. In turn, students would be valued as active partakers in the process of coming to know rather than passive assimilators of codified information. Thus, knowledge would no longer be pursued as objective bits of information, for knowledge would be inseparable from the learner, the teacher, the school, the books, and so on. 'Inter-being' or 'mutuality' sums up the first principle of participatory ethics and a corollary principle of moral education.

\section{Patterns that Connect}

The above first principle outlines an orientation and approach to the world: through attitude and awareness of Inter-being. The second principle shall address how to work with Inter-being. Returning to our earlier tract about patterns, in participatory ethics, what we have to work with primarily are patterns - patterns that connect. But what are these patterns? How do they show up for ethics? 
Complexity theory discusses "fractals" and "butterfly effects" ${ }^{25}$ Fractal refers to "a shape that is recursively constructed or self-similar, that is, a shape that appears similar at all scales of magnification and is therefore often referred to as "infinitely complex". ${ }^{26}$ Nature abounds with fractal images. Natural objects and phenomena, such as mountains, coastal lines, clouds, snowflakes, a tree branch, a frond from a fern or blood vessels, display self-similar structure over an extended, but finite, scale of range. A key notion of fractals is that of self-similarity, which is different from identicalness. Self-similarity results from taking a simple shape, introducing to it a simple rule of alteration and iterating, that is, recursively repeating, the operation at different scales or magnitude. This operation results in repetition of pattern ad infinitum. Computer-generate fractal images graphically illustrates this operation. These images are so natural to us that they echo a stirring deep within when we view them, for they are the patterns we phenomenal beings embody. Fractals are us. This sort of realization leads us to see and feel the phenomenal world as infinite reiterations of selfsimilarity. All we see, hear, and touch reminds us that we are, the whole world is, intricate and complex variations of the same theme: Life. Snowflakes falling through calm of night and resting ever so briefly upon warm skin reminds us of our inter-being with air, water, clouds, sky, other sentient beings' breaths, in short, everything around us. Immersing deeply in, becoming one with, such experience takes us to participatory knowing: snowflakes are us. As educators we can bring this sense of inter-beingness of Life to our practice and to our students. Most basically this plays out daily in how we accept one another as self-similar beings across their differences. This self-similarity can run right through the curriculum and helps us to see and appreciate the nature of inter-being that we embody, that we are. The experience of inter-being through perception of self-similarity does not deny the uniqueness of the individual beings based on difference. In inter-being, difference is not objectified, and leads to a sense of alienation. Difference in inter-being does not breed opposition but invites continual pattern-making of self-similarity. The whole phenomenal world, of which we are an integral part, is but an inexhaustible play of self-similarity.

Another complexity concept we can work with is the butterfly effect in chaotic dynamics, or more technically known as the "sensitive dependence on initial conditions." This effect recounts influential relationality by allowing contemplation of origin and interaction as initial conditions become amplified to the point where predictability becomes impossible. Within this unknowing lies a vast terrain of creative possibilities in generous influence and confluence. While in deontological models a source can and is identified, complexity's recollection of wings stirring pan-Pacific storms shifts focus from nexus (that is, discrete and autonomous objects) to relationship--the sphere of influence and confluence. Apply this idea to human development and change. As educators, we can never know how minute or insignificant responses we make to others 
will impact them and their future development. A slightly rude response we have carelessly given to a child might go straight to her heart and cause a great pain that affects her emotional development in an adverse way. Or, it can work the positive way, too. Thus contemplating the butterfly effect, we shift focus from the narrow view of figuring out the sequence and consequence of linearly conceived responsibility to creative and generous potentials inherent in relationship-that is, in patterns that connect and amplify. ${ }^{27}$

\section{Concluding Reflection}

If singularity of source is unattainable, and we are all reiterations of one another, then what are ethics? What is moral education? From the complexity perspective, to become morally educated is to remember our inter-beingness, care-fullness and generosity. It is to arouse in each other recollections of inter-being. Fractals and all patterns that connect are markers, reminding us of our inter-beingness. Also, we need to exercise care and carefulness in our dealings with, and responses to, the world since we know neither the kind nor the proportion of our actions' future. Our ignorance should motivate us to be not only especially cautious and concerned, but also to act in extra generous ways to invite good chance. Like a farmer who sows more seeds than necessary, just in case many do not germinate, one should strive to do more than what is conventionally obligatory as ethical. Moral education from the complexity perspective is not primarily about instilling or imparting correct and "moral" (as opposed to immoral) beliefs and values, nor about "doing the right thing" and behaving morally (respectfully, fairly, and so on), but about coming into the consciousness of inter-beingness through immersing oneself in the complexity phenomena of the world. Earlier, we briefly explored two principles of such phenomena: fractals and butterfly effect. For moral educators informed by complexity views, the present day preoccupation with accountability and even social responsibility, and moral virtues, moral reasoning and behaviour, all indicate narrow focus on discrete individuals (rather than complex, interpenetrating presences) and short-sighted concerns with codes, rules and consequence. The result tends to be moralism: shoulds and shouldn'ts, rights and wrongs, directed at individuals, especially ones who are seen as transgressive and abberant. Insight from complexity views steers us away from moralism, and takes us the holistic vision of inter-being--to complexity. Does William Blake, then, pen both lines of poetry and moral education?

To see a World in a Grain of Sand

And Heaven in a Wild Flower

Hold Infinity in the palm of your hand

And Eternity in an hour. ${ }^{28}$ 


\section{Notes}

1. There is always the gap between 'ought' and 'is' (naturalistic fallacy). 'Ought' is not what is. In conceiving of ethical ways inspired by complexity, we the authors depart from this conventional and traditional normative understanding of ethics, namely, morality, and reach for a way of ethics that works with 'what is', that is, whatever is at hand, here and now, each moment. As we shall illustrate in this paper, it is by virtue of participating deeply in the 'is-ness', not in the 'ought-ness', that we can change reality and ourselves.

2. Bateson (1980) in discussing the feel of culture explores the notion of 'ethos' as feeling. This understanding is inherent in the etymology of 'ethics' as its Greek root goes to 'ethos', the habits of heart and mind, that is, a way of being or life, permeating and characterizing a group.

3. We the authors confess our difficulty in conjuring up an amenable audience as we write this paper that pulls together many disparate areas of thought and research, and explain concepts and theories, each of which would require, for many readers' better comprehension, a more extended treatment than we can supply here. What this may mean is frustration for many readers. As well, we face the self-imposed challenge of writing in such a style that "medium is message." Nonetheless, our hope is that there is much in this writing to evoke and provoke thoughts and feelings towards, not to mention taste for, the way or the Dao of complexity.

4. Thompson (1996).

5. Lawrence Kohlberg's (1971) influential theory, Stages of Moral Development, presupposed this gender bias. Carol Gilligan (1982) critiqued and reframed this work to demonstrate gender difference in moral orientation, rejecting the assumption of inferiority of female moral development.

6. Textual evidence locates the inauguration date of a new worldview to the $17^{\text {th }}$ century, one filled with arguments for a universe composed of inanimate matter, characterized as objective, measurable, quantifiable, and ruled by laws of mechanics.

7. Laing's (1980) incisive summary of mental processes involved in transforming subjective into objective describes: "(1) the ablation or elimination of some or all sense data; (2) the temporary suspension of any subject of cognizance; (3) the cutting off of any relation of intersubjectivity, or interiority; (4) the de-realization of any subjectivity out there" (p. 9).

8. In contesting the picture of a deterministic universe, we are not arguing for indeterminism understood as the thesis that there are no perceivable or probable patterns of cause and effect or that we cannot know such patterns. Our challenge to determinism is disputation concerning linear causality, meaning that the world is governed by discrete, explicate and linear chains of determining, that is, formative influence. We see that not only the directions and reaches but also the nature of influence that shape beings and their events are non-discrete and nonlinear, hence, complex, which renders beings and events themselves in likewise manners. In this situation, probable patterns and emergent outcomes are all we can work with. We can hence speak of our universe being underdetermined, not undetermined. We beg our readers to keep in mind the distinction we are making here among determinism, indeterminism, and under-determinism, and we also ask them to note that we are not arguing for indeterminism in our contestation of a deterministic universe.

9. Capra (1975); Berman (1989). 
10. Varela (1992, p. 104)

11. To explore this more philosophically and psychologically, see Buber's (1996) distinction between 'I- Thou' and 'I-It', the latter being this objectified relationship. Also see Bai (2001).

12. In contrasting deontology to naturalism, and in implying, as we do here, an endorsement of the latter over former, we are not further implying (as some may read us) that we should not have duties and obligations or make them a moral motivation. It is "natural" and "good" to have duties and feel obliged. What becomes very problematic is when duties and obligations become the main or the sole moral motivation to act, and their "imperatives" are set against, and over and above, one's inclination and sympathetic resonance.

13. According to the ontology of the relational universe (embedded universe, implicate universe, mutual universe), that we are all embedded in each other's reality is a statement of "fact," whether we know it or not. However, it is not until one realizes this, not just intellectually but experientially, that one is able to live the ethical life of relationality or 'inter-being', to borrow Hahn's $(1992,1998)$ expressive neologism.

14. We are not saying that individuals separately and consciously choose to live, let alone construct, a universe. Rather, individuals, being part of the collective with a shared history, inherit a particular universe (world) and continue to unconsciously enact and re-enact it, thereby perpetuating its existence. See Bourdieu (1977).

15. The insight in this paragraph takes us to an inquiry into the history of western philosophy, starting with Plato and his Noumenal world, passing through Descartes' quest for certainty through mathematization of the phenomenal world, and down to the contemporary state of artificial intelligence's pursuit of the perfection of limited natural human intelligence. We cannot pursue this inquiry in this paper, but its mention gives a sense of the immense philosophical backdrop against which we are exploring connections among psychology, ethics, and ontology.

16. Capra (1996); Macy (1991).

17. Nicolis \& Prigogine (1977).

18. Here we are reminded of Zen and its ways of interrupting the conceptual operations of the dualistic, objectifying mind. Zen has developed diverse arts, from flower arrangement to martial arts, which take us out of the confines of the dualistic conceptual mind into to the vastness of non-dual, non-discursive, and non-objectifying consciousness.

19. Claxton (1980). Of course, we are not implying that all mathematical thinking is linear, logical, analytic, and mathematicians think only linearly, and so on. Far from the truth.

20.Skolimowsk (1995).

21. Giddens (2003), op. cit., prophetically pronounced that the most urgent and critical problem the globalized world faces today is the rise of fundamentalism.

22. Note, though, that rules, maxims, and the like need not be, and are not always used as, devices of control and manipulations. True, they most often are, but they can be utilized differently, as heuristic learning and teaching tools that are consequently adopted by people for the purpose of each person cultivating a certain quality of being with himself/herself. Thus, use does determine the meaning of something, to affirm the spirit and tenet of Pragmatism.

23. Bai \& Chinnery (2006) expose the psychology of domination and control as part of the background political thought behind the social contract. 
24. Hahn (1992)

25. Gleick (1987), Capra (1996).

26. http://en.wikipedia.org/wiki/Fractal

27. Capra, F. op. cit.

28. Blake, W. From Auguries of Innocence, c. 1800.

\section{References}

Bai, H. 2001. Challenge for education: Learning to value the world intrinsically. Encounter 14(1): 4-16.

Bai, H. and Chinnery, A. 2006. Justice in the name of the other: Levinas on rights and responsibility. In D. Egea-Kuehne (Ed.). Levinas and education: At the intersection of faith and reason. London: Routledge.

Bateson, G. 1980. Steps to an ecology of mind. New York: Ballantine.

Bateson, G. 2002. Mind and nature: A necessary unity. New York: Hampton.

Berman, M. 1989.Coming to our senses: Body and spirit in the hidden history of the West. New York: Simon \& Schuster.

Blake, W. 1977. The portable Blake. New York: Viking.

Bourdieu, P. 1977. Outline of a theory of practice. Cambridge, UK: Cambridge University Press.

Buber, M. 1996. I and Thou. New York: Touchstone.

Capra, F. 1975. The Tao of physics. Boston: Shambhala.

Capra, F. 1996. The web of life: A new scientific understanding of living systems. New York: Anchor Books.

Claxton, G. 1997. Hare brain, tortoise mind. New York: The Ecco Press.

Giddens, A. 2003. Runaway world: How globalization is reshaping our lives. New York: Routledge.

Gilligan, C. 1982. In a different voice. Cambridge, MA: Harvard University Press.

Gleick, J. 1987. Chaos: Making of a new science. New York: Viking.

Hahn, T.N. 1992. Peace is every step: The path of mindfulness in everyday life. New York: Bantam Books.

Hahn, T.N. 1998. Interbeing: Fourteen guidelines for engaged Buddhism. Berkeley, CA: Parallax Press.

Juarrero, A. 1999. Dynamics in action. Cambridge, MA: MIT.

Laing, R.D. 1980. What is the matter with mind? In S. Kumer (Ed.). The Schumacher Lectures (pp. 1-27). London: Sphere Books.

Macy, J. 1991. Mutual causality in Buddhism and general systems theory. New York: State University of New York Press.

Nicolis, G. and Prigogine, I. 1977. Self-organization in non-equilibrium systems. New York: Wiley.

Skolimowsk, H. 1995.The participatory mind: A new theory of knowledge and of the universe. London: Arkana, Penguine.

Thompson, W.I. 1996. Coming into being: Artifacts and texts in the evolution of consciousness. New York: St. Martin's Griffin.

Varela, F. 1999. Ethical know-how: Action, wisdom, and cognition. CA: Stanford University Press. 


\begin{abstract}
About the Authors
Heesoon Bai is an Associate Professor in the Faculty of Education at Simon Fraser University. She can be reached at hbai@sfu.ca and www.educ.sfu.ca/fri/hbai. Hartley Banack is a doctoral student in the Faculty of Education at Simon Fraser University. He can be reached at hbanack@sfu.ca.
\end{abstract}

(c) Copyright 2006. The authors, Heesoon Bai and Hartley Banack assign to the University of Alberta and other educational and non-profit institutions a non-exclusive license to use this document for personal use and in courses of instruction provided that the article is used in full and this copyright statement is reproduced. The authors also grant a non-exclusive license to the University of Alberta to publish this document in full on the World Wide Web, and for the document to be published on mirrors on the World Wide Web. Any other usage is prohibited without the express permission of the authors. 